\title{
ANÁLISE DO IMPACTO DA CIRURGIA BARIÁTRICA EM UMA POPULAÇÃO DO CENTRO DO ESTADO DO RIO GRANDE DO SUL UTILIZANDO O MÉTODO BAROS
}

\author{
Carlise Felkl PREVEDELL0¹, Elisângela COLP0¹, Elveni Teresinha MAYER² e Hairton COPETTI ${ }^{3}$
}

RESUMO - Contexto - Devido às alterações metabólicas causadas pelo excesso de peso, a cirurgia bariátrica tem sido muito utilizada, a fim de proporcionar perda e manutenção de peso a longo prazo na população com obesidade mórbida. Objetivo - Analisar o impacto da cirurgia bariátrica em uma população do centro do estado do Rio Grande do Sul utilizando o método BAROS. Métodos - Foi realizado estudo retrospectivo, com 32 pacientes, de ambos os sexos, entre 19 e 44 anos de uma clínica particular de Santa Maria, RS. Analisaram-se as variações de peso e índice de massa corpórea, hábito intestinal, uso de tabaco e prática de atividade física para comparar resultados nos períodos de pré e pós-operatório. Os pacientes foram divididos em: grupo A (pré-operatório), grupo B (até 6 meses de pós-operatório), grupo C ( 7 a 18 meses de pós-operatório) e grupo D (19 a 30 meses de pós-operatório). Para avaliação da eficácia da cirurgia bariátrica foi utilizado o método BAROS. Para análise estatística foi utilizado o teste ANOVA uma via e post-hoc seguido de Duncan's. Foram considerados estatisticamente significantes os dados com $P<0,05$. Os resultados foram expressos em média \pm EP. Resultados - Os pacientes apresentaram redução significativa no peso, no pré-operatório. A média foi de $118,8 \mathrm{~kg}( \pm 2,89)$, e no pós-operatório, o grupo B apresentou $89 \mathrm{~kg}( \pm 5,69)$, o grupo C 76,8 $\mathrm{kg}( \pm 4,85)$ e o grupo D $76 \mathrm{~kg}( \pm 4,46)$, demonstrando perda de peso gradativa. Do mesmo modo, o índice de massa corpórea apresentou redução significativa nos grupo B, C e D, em relação ao grupo A. Os pacientes apresentaram melhora do hábito intestinal de 15,6\% no pós-operatório em relação ao pré-operatório. Quanto ao uso de tabaco, houve redução de 33,3\% no pós-operatório. A prática de atividade física aumentou em 34,4\% entre os pacientes após a cirurgia. De acordo com a aplicação do método BAROS, os pacientes apresentaram classificações excelente, muito bom e bom, não apresentando classificação insuficiente. Conclusão - De acordo com os resultados do método BAROS e das características avaliadas dos pacientes, pode-se observar que a cirurgia bariátrica apresentou efeitos satisfatórios nessa população. DESCRITORES - Obesidade mórbida. Perda de peso. Gastroplastia. Qualidade de vida.

\section{INTRODUÇÃO}

A obesidade é hoje um grave problema de saúde pública que atinge praticamente o mundo todo, sobretudo os países desenvolvidos e em desenvolvimento. O aumento da prevalência de obesidade em várias regiões do planeta vem se revelando como um dos mais importantes fenômenos clínico-epidemiológicos da atualidade, independente de condições econômicas e $\operatorname{sociais}^{(3,22,23,29)}$.

A obesidade é uma doença crônica universal, caracterizada pelo acúmulo excessivo de gordura corporal. Trata-se de fenômeno multifatorial que envolve componentes genéticos, comportamentais, psicológicos, sociais, metabólicos e endócrinos ${ }^{(7,8,11,23)}$.

A obesidade grave é uma das doenças que mais matam no mundo em decorrência de suas comorbidades, estando na lista das 10 doenças mais mortais do planeta. É o fator de risco mais importante para o desenvolvimento de diabetes mellitus tipo 2, além disso está relacionada com o desenvolvimento da hipertensão arterial sistêmica (HAS), insuficiência cardíaca congestiva, dislipidemia e aterosclerose, contribuindo, assim, para maior risco de morbimortalidade por doenças cardiovasculares. Também é responsável por desencadear artropatias, hipoventilação ou síndrome da apneia do sono e muitas outras condições patológicas, reduzindo, assim, a qualidade de vida e a auto-estima do paciente. Devido a todas comorbidades associadas à obesidade, somandose os aspectos emocionais abalados, pacientes muito obesos têm taxa de mortalidade até 12 vezes maior que a população em $\operatorname{geral}^{(2,4,5,7,11,13,15,24,29)}$.

A obesidade também pode estar associada a maior incidência de complicações cirúrgicas e obstétricas,

Centro Universitário Franciscano (UNIFRA), Curso de Nutrição em convênio com a Clínica Cirúrgica do Aparelho Digestivo, Santa Maria, RS.

${ }^{1}$ Centro Universitário Franciscano, Santa Maria, RS; ${ }^{2}$ Universidade do Contestado, Concórdia, SC; ${ }^{3}$ Universidade Federal de Santa Maria, RS.

Correspondência: Nutr. Elisângela Colpo - Rua Tupinambá, 58 - Camobi km 7 - 97110-620 - Santa Maria, RS. E-mail: elicolpo@unifra.br 
e maior suscetibilidade a acidentes. Apesar da obesidade não estar associada ao aumento de risco em problemas psiquiátricos, esses pacientes têm maior propensão a problemas econômicos, sociais e psíquicos e, muitas vezes, sentem-se discriminados na vida pessoal e profissional. Devido a isto, esta doença tem sido apontada como um dos fatores contribuintes para a baixa-estima, o isolamento social e a depressão ${ }^{(6,8,9)}$.

Neste contexto, os pacientes com obesidade grave devem ser encarados como pessoas que possuem menos qualidade de vida e condições clínicas que podem levá-las a morte. Por estes motivos, são necessárias abordagens eficientes para promover significativa redução de peso. A orientação dietética, a programação de atividade física e o uso de fármacos antiobesidade são os pilares principais do tratamento. Porém, a terapia clínica tem sucesso limitado a curto prazo e quase inexistente a longo prazo ${ }^{(13,27)}$. De acordo com SEGAL e FANDIÑO ${ }^{(29)}$ não há manutenção na perda de peso, pois cerca de $95 \%$ dos pacientes recuperam seu peso inicial em até 2 anos de tratamento convencional.

A indicação do tratamento cirúrgico deve basear-se numa análise abrangente de múltiplos aspectos clínicos. A avaliação desses pacientes no pré e pós-operatório deve ser realizada por equipe multidisciplinar. São candidatos ao tratamento cirúrgico pacientes com o IMC maior que $40 \mathrm{~kg} / \mathrm{m}^{2}$ ou com IMC superior a $35 \mathrm{~kg} / \mathrm{m}^{2}$ associado à comorbidades, fracasso de métodos conservadores de emagrecimento bem conduzidos, condições psicológicas para cumprir a orientação dietética no pós-operatório e ausência de causas endócrinas de obesidade ${ }^{(5,12,18,27)}$.

Para avaliar a eficiência e impacto da cirurgia bariátrica na qualidade de vida destes pacientes é importante utilizar métodos padronizados e respeitados. Entre os muitos conhecidos, pode-se destacar o de BAROS (Bariatric Analysis and Reporting Outcome System) de ORIA e MOOREHEAD ${ }^{(20)}$, método avaliado e reconhecido internacionalmente, prático e eficiente para a estimativa dos resultados do tratamento cirúrgico da obesidade mórbida, pois se baseia na análise de importantes aspectos que devem se modificar na saúde e na vida dos pacientes pós-cirurgia bariátrica: a perda do excesso de peso, melhora da comorbidez e da qualidade de vida e também de eventuais complicações da cirurgia ${ }^{(11,19,20)}$.

Devido ao aumento de procedimentos cirúrgicos para promover a perda de peso, nos dias atuais, o objetivo deste trabalho foi analisar o impacto da cirurgia bariátrica em uma população do centro do Estado do Rio Grande do Sul utilizando o método BAROS.

\section{MATERIAL}

Foi realizado estudo retrospectivo no período de março a junho de 2007, em pacientes que realizaram cirurgia bariátrica de acordo com as técnicas de bypass gástrico e derivação biliopancreática em uma clínica particular de Santa Maria, RS. Participaram do estudo 32 pacientes com evolução cirúrgica de 6 a 30 meses de pós-operatório. Todos assinaram o termo de consentimento livre e esclarecido com autorização de seus dados, após o projeto de pesquisa ser aprovado pelo comitê de ética do Centro Universitário Franciscano com o número de protocolo 218.2006.2.

\section{Métodos}

Por meio de visitas periódicas na clínica e nas residências dos pacientes, foram realizadas entrevistas e análise do prontuário médico. Durante as entrevistas, foram aplicados os questionários sobre a qualidade de vida no pós-operatório (PO) de cirurgia bariátrica, segundo ORIA e MOOREHEAD ${ }^{(20)}$, e aferido o peso atual, com balança de uso pessoal, para posterior cálculo da percentagem de perda que faz parte da aplicação do método BAROS.

A perda de peso foi avaliada através das diferenças dos pesos e IMC no pré e pós-operatório, como também o percentual de perda do excesso de peso (\%PEP) no pósoperatório. Os IMCs foram classificados conforme a tabela adaptada da $\mathrm{OMS}^{(30)}$.

Dados de peso e altura foram obtidos da avaliação nutricional pré-cirúrgica. Durante a entrevista, os pacientes foram questionados sobre o hábito intestinal, consumo de tabaco, prática de atividade física, uso de medicação para perda e peso, entre outros.

Depois dos dados compilados, as informações foram analisadas através da pontuação das tabelas e do questionário integrantes do método BAROS. De acordo com o preenchimento destas tabelas e do questionário, os pacientes receberam uma pontuação, sendo que as complicações e as reoperações reduziam pontos. Conforme sua pontuação final, os pacientes foram enquadrados em um dos grupos que recebeu a denominação adequada a sua evolução cirúrgica: insuficiente, aceitável, bom, muito bom ou excelente.

Os 32 pacientes foram divididos em três grupos: grupo B (até 6 meses de PO), grupo C (de 7 a 18 meses de PO) e grupo D (de 18 a 30 meses de PO), a fim de traçar um paralelo comparativo destes três grupos conforme sua evolução cirúrgica no pós-operatório em relação ao grupo controle, que são os mesmos pacientes no pré-operatório (grupo A).

Os resultados foram expressos em médias $(\bar{X})$ e erropadrão (EP) e comparados nos diferentes grupos de evolução cirúrgica e no grupo controle (pré-operatório) através do software Statistic 6.0., utilizando os testes ANOVA uma via e Post-Hoc seguido de Duncan's, além de Medidas Repetidas quando necessário. Foram considerados estatisticamente significativos os dados com $P<0,05$.

\section{RESULTADOS}

Em relação aos 32 pacientes submetidos a cirurgia bariátrica, $75 \%$ foram do sexo feminino e $25 \%$ do sexo masculino. A idade variou de 19 a 44 anos, com média de 32,4 anos. A caracterização étnica, o estado civil e a escolaridade dessa população estão dispostos na Tabela 1 .

De acordo com a Figura 1, pode-se observar que $68,8 \%$ da população apresentava hábito intestinal normal anteriormente à cirurgia. Após a cirurgia, os pacientes tiveram melhora em relação a constipação, aumentando o hábito intestinal normal 
para $84,4 \%$ no pós-operatório. Já a diarreia permaneceu com índices de $6,25 \%$.

TABELA 1. Caracterização dos obesos submetidos a cirurgia bariátrica em uma clínica de Santa Maria, RS

\begin{tabular}{lccc}
\hline $\begin{array}{l}\text { Estado nutricional } \\
(\%)\end{array}$ & $\begin{array}{c}\text { Raça } \\
(\%)\end{array}$ & $\begin{array}{c}\text { Estado civil } \\
(\%)\end{array}$ & $\begin{array}{c}\text { Escolaridade } \\
(\%)\end{array}$ \\
\hline obesidade grau III (78) & brancos (94) & solteiros (53) & $3^{\circ}$ grau completo (29) \\
obesidade grau II (22) & negros (3) & casados (38) & $\begin{array}{c}3^{\circ} \text { grau incompleto (26) } \\
\text { mulatos (3) }\end{array}$ \\
& & divorciados (6) & $2^{\circ}$ grau completo (23) \\
& & viúvos (3) & pós-graduação (19) \\
& & especialização (3) \\
\hline
\end{tabular}

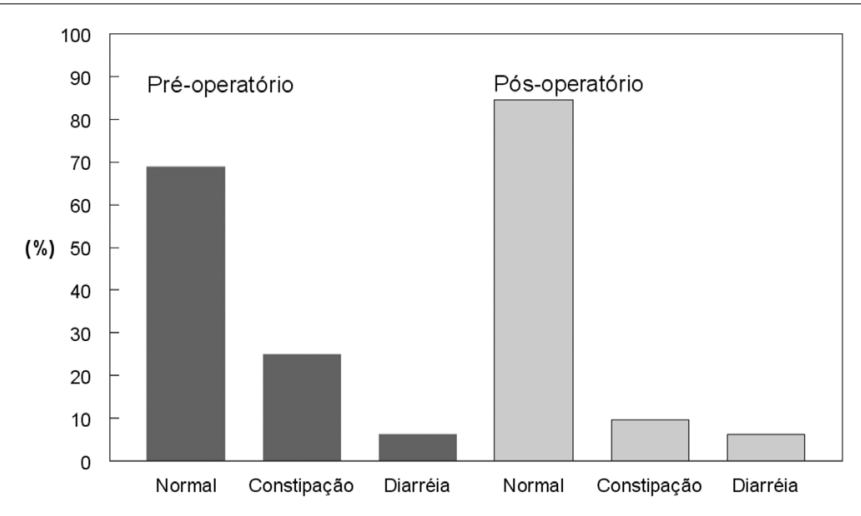

FIGURA 1. Hábito intestinal de pacientes no pré e pós-operatório de cirurgia bariátrica em uma clínica de Santa Maria, RS

Quanto ao uso do tabaco (Figura 2), após a cirurgia houve diminuição do seu consumo, pois dos seis pacientes que apresentavam esse hábito, dois deixaram de fumar, ou seja, $33,3 \%$.

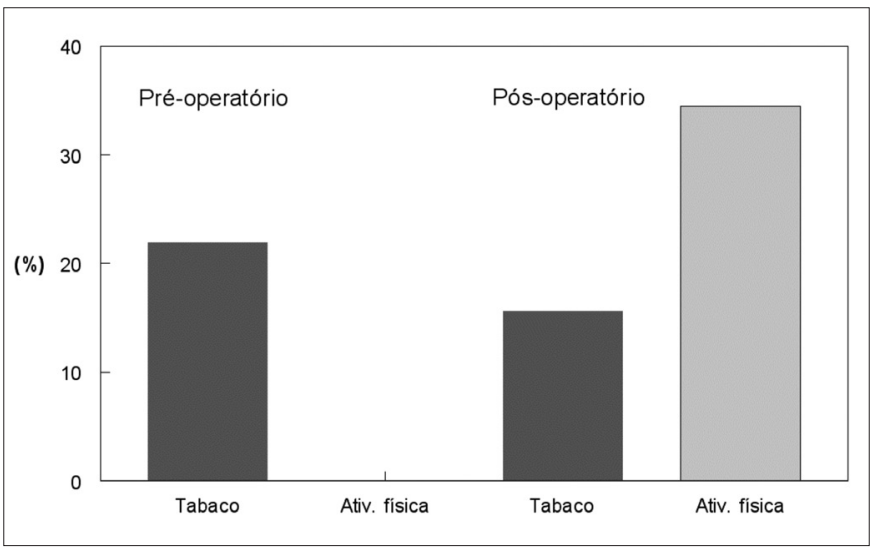

FIGURA 2. Uso de tabaco e prática de atividade física de pacientes no pré e pós-operatório de cirurgia bariátrica em uma clínica de Santa Maria, RS

Em relação à prática de atividade física, nenhum dos pacientes relatou estar realizando-a regularmente antes da cirurgia. Após o procedimento cirúrgico, $34,4 \%$ dos pacientes realizavam atividade física no momento da entrevista, conforme
Figura 2. Outra relação constatada foi o percentual de realização de atividade física entre os grupos: no grupo B, $62,5 \%$ dos entrevistados realizavam atividade física regularmente, enquanto no grupo C foi demonstrado $36,4 \%$ e no grupo D apenas $15,4 \%$, demonstrando que conforme o período de pós-operatório e quanto mais próxima a perda de peso do ideal desejado, maior é o desinteresse dessa população pela prática de atividade física.

Em relação ao peso dos pacientes, no pré-operatório (grupo controle) houve média de $118,8 \mathrm{~kg}( \pm 2,89)$, no grupo B $89 \mathrm{~kg}( \pm 5,69)$, no grupo C $76,8 \mathrm{~kg}( \pm 4,85)$ e no grupo D $76 \mathrm{~kg}( \pm 4,46)$, conforme a Figura 3 , demonstrando perda de peso gradativa de acordo com o tempo pós-cirúrgico e estabilização desse peso próximo aos 19 meses de cirurgia.

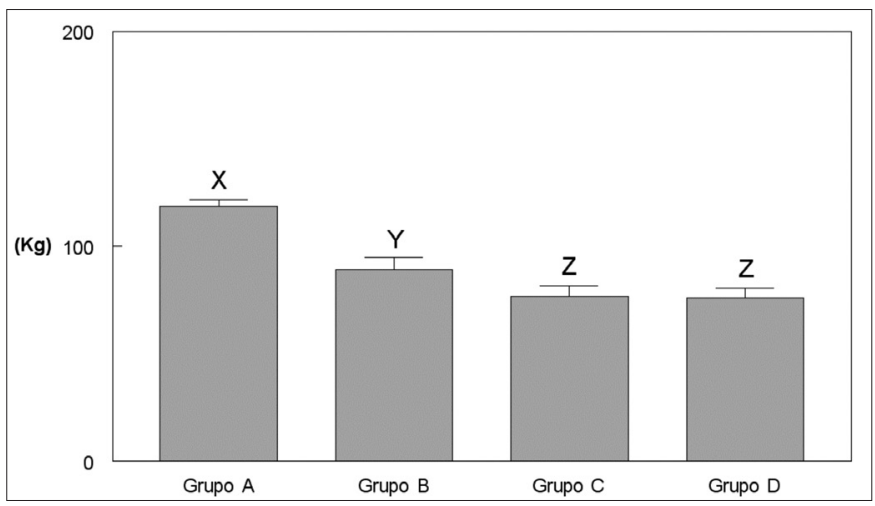

FIGURA 3. Médias dos pesos $(\mathrm{kg})$ de pacientes no pré e pós-operatório de cirurgia bariátrica em uma clínica de Santa Maria, RS. As letras X, Y e $Z$ representam variações estatisticamente significantes $(P<0,05)$. Teste ANOVA uma via seguido de Duncan's

As médias observadas dos IMCs (Figura 4) foram de 43,6 $\mathrm{kg} / \mathrm{m}^{2}( \pm 0,9)$ no grupo controle, $34,3 \mathrm{~kg} / \mathrm{m}^{2}( \pm 1,78)$, no grupo B, $27,3 \mathrm{~kg} / \mathrm{m}^{2}( \pm 1,51)$ no grupo C, e no grupo D $28,4 \mathrm{~kg} / \mathrm{m}^{2}$ $( \pm 1,39)$, evidenciando queda significativa e gradativa do IMC até o $18^{\circ}$ mês e após ganho de massa corpórea no grupo D.

O \%PEP teve média total de $55 \%( \pm 2,66)$, sendo $42 \%$ $( \pm 2,91)$ no grupo $\mathrm{B}, 57,7 \%( \pm 3,32)$ no grupo $\mathrm{C}$, e $64,9 \%$

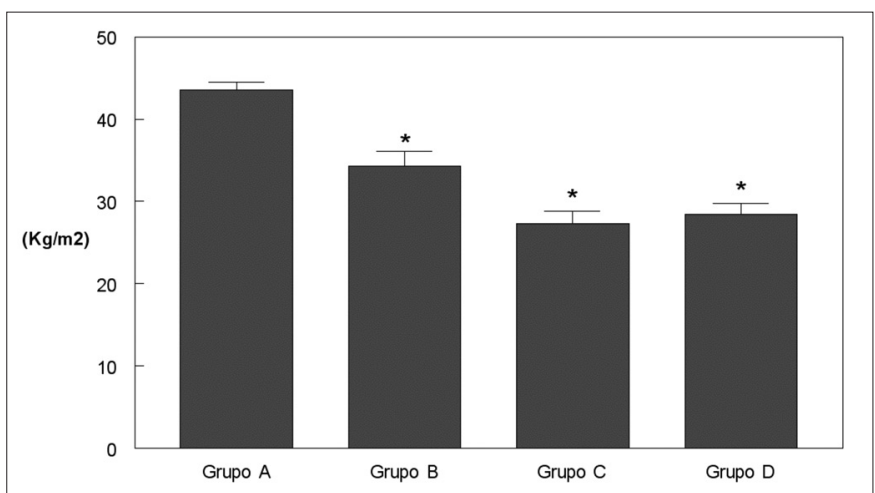

FIGURA 4. Médias dos IMCs $\left(\mathrm{kg} / \mathrm{m}^{2}\right)$ de pacientes $(\mathrm{n}=32)$ no pré e pós-operatório de cirurgia bariátrica em uma clínica de Santa Maria, RS $* P<0,05$ em relação ao grupo A (ANOVA medidas repetidas) 
$( \pm 4,21)$ no grupo $\mathrm{D}$, evidenciando perda de peso gradativa de acordo com o período de pós-operatório.

De acordo com a aplicação do método BAROS, os pacientes que participaram da pesquisa tiveram a seguinte classificação: excelente $(21,9 \%)$, muito bom $(50 \%)$, bom $(28,1 \%)$ e nenhum dos pacientes teve evolução aceitável ou insuficiente (Figura 5).

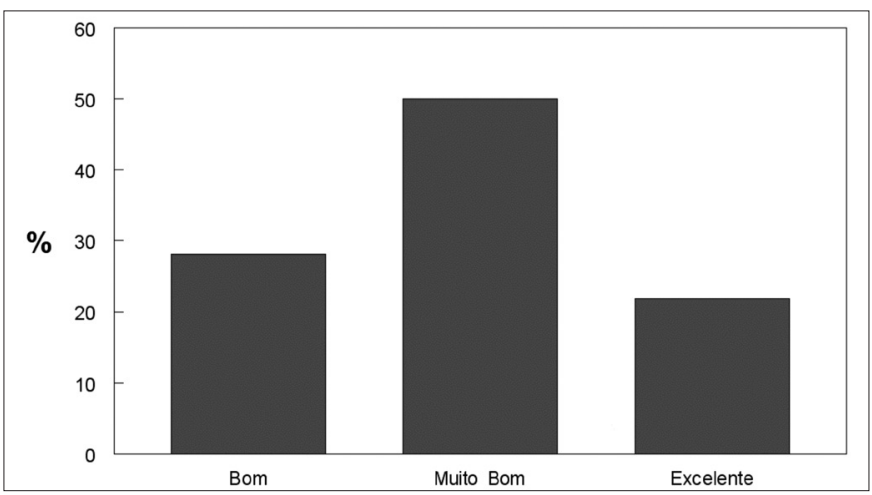

FIGURA 5. Classificação do método BAROS (\%) pós-operatório de cirurgia bariátrica em uma clínica de Santa Maria, RS

\section{DISCUSSÃO}

O predomínio do sexo feminino na população pode ser entendido pela citação de MAZZEO et al. ${ }^{(18)}$, em que mulheres de todos os grupos étnicos possuem maior possibilidade do que homens em submeter-se a cirurgia bariátrica. Isto pode estar relacionado a maior motivação das mulheres obesas do que os homens obesos em perder peso, talvez em consequência das pressões a respeito da estética. No estudo "The worldwide obesity epidemic", JAMES et al. ${ }^{(17)}$ afirmaram que na maioria dos países, as mulheres possuem distribuição maior de IMC e obesidade que os homens, justificando assim, sua maior procura pela cirurgia bariátrica.

De acordo com os resultados do presente estudo pode-se relatar que a cirurgia bariátrica contribuiu para a melhora do hábito intestinal, o aumento da prática de atividade física e a diminuição do uso do tabaco nessa população.

Analisando os resultados do estudo de QUADROS et al. ${ }^{(25)}$, a diarreia e a constipação estiveram presentes entre os pacientes no pós-operatório em percentagens pequenas e menores que no presente estudo, respectivamente $4,24 \%$ e $2,42 \%$. A diarreia pode ser explicada devido ao consumo excessivo de carboidratos simples (resultado da síndrome de dumping) e alimentos gordurosos, associados à exclusão do duodeno e a maior parte do jejuno proximal. Esta manifestação pode ser amenizada pelo incentivo ao controle dietético e à reeducação alimentar no pós-operatório.

Em estudo de REIS et al. ${ }^{(26)}$, ocorreu diminuição de 50\% do uso do tabaco, maior que no presente estudo que foi de $33,3 \%$. No mesmo estudo, analisando a prática de atividade física no pré e pós-operatório, houve aumento de $28,6 \%$ da prática de atividade física no pós-operatório, dado menor que o encontrado na presente série que foi de $34 \%$.
De acordo com FUJIOKA ${ }^{(10)}$, em 18 a 24 meses após a cirurgia bariátrica, quase todos os pacientes declinaram a perda de peso e por consequência mantiveram ou passaram pelo período de reganho de peso. HSU et al. ${ }^{(16)}$ descreveram perda de peso em $30 \%$ dos pacientes, passando por seu aumento posteriormente.

De acordo com estudo realizado na Faculdade de Medicina da Universidade de São Paulo ${ }^{(12)}$, o peso médio no préoperatório de pacientes que se submeteram a gastroplastia vertical com derivação gastrojejunal em Y-de-Roux foi de $142,6 \mathrm{~kg}$, após 6 meses $104 \mathrm{~kg}$, até 12 meses $88,6 \mathrm{~kg}$ e de 18 a 24 meses 90,4 kg. As médias de peso confirmam a perda de peso gradativa e demonstram o reganho após 18 meses de cirurgia, fato não observado neste estudo.

As variações não significativas de peso nos grupos $\mathrm{C}$ e D podem ser explicadas, de acordo com a "American Society for Metabolic \& Bariatric Surgery"(1), em que é justamente o momento do paciente reestabilizar a massa magra do corpo apropriada ao peso corporal total.

No estudo de PARADIS et al..$\left.^{(21}\right)$ encontrou-se IMC préoperatório de $56,7 \mathrm{~kg} / \mathrm{m}^{2}( \pm 4,2)$, consequentemente, o IMC até 6 meses de $\mathrm{PO}$ teve média $40,3 \mathrm{~kg} / \mathrm{m}^{2}( \pm 3,5)$, resultados mais elevados que no presente estudo. Porém, o resultado do IMC até 24 meses de PO teve o mesmo resultado deste estudo, que compreende os 24 meses de PO, ou seja, 28,4 $\mathrm{kg} / \mathrm{m}^{2}( \pm 1,4)$. Pode-se observar nestes estudos que quanto maior o tempo de $\mathrm{PO}$, maior a dificuldade de perda de peso.

Em estudo publicado no Chile por GONZÁLEZ et al. ${ }^{(14)}$, com 31 pacientes submetidos a bypass gástrico com mais de 12 meses de PO, o resultado observado da avaliação da cirurgia bariátrica, de acordo com o escore de BAROS, foi excelente em $58,1 \%$ dos pacientes, muito bom em $22,6 \%$, bom em $16,1 \%$, e $3,2 \%$ aceitável, não tendo nenhum paciente com evolução insuficiente. Já em estudo brasileiro, FARIA et al. ${ }^{(8)}$ avaliaram 160 pacientes com obesidade mórbida que foram submetidos a gastroplastia redutora com bypass gástrico em Y-de-Roux com anel de contenção (operação de Fobi-Capella) nos anos de 2000 a 2002, obtendo os seguintes resultados: excelente $60,6 \%$, muito bom $26,8 \%$, bom $9,8 \%$ e ruim $2,8 \%$. Resultados mais satisfatórios foram encontrados no presente estudo, de acordo com o método BAROS.

\section{CONCLUSÕES}

A evolução de perda de peso desta população foi gradual, de acordo com os tempos de PO, fato também observado em outros estudos nacionais e internacionais consultados. Porém, estes pacientes não apresentaram reganho de peso após 18 meses de PO como na maioria dos estudos, possivelmente pela prática de atividade física observada.

Os resultados do método BAROS destacaram a eficiência da cirurgia nesta população, pois os pacientes apresentaram classificações excelente, muito bom e bom, não apresentando classificação insuficiente. Somando estes dados com a melhora do hábito intestinal e prática de atividade física, pode-se observar que a cirurgia bariátrica apresentou efeitos satisfatórios nessa população. 
Prevedello CF, Colpo E, Mayer ET, Copetti H. Analysis of the bariatric surgery impact in a population from the center area of Rio Grande do Sul State, Brazil, using the BAROS method. Arq Gastroenterol. 2009;46(3):199-203.

ABSTRACT - Context - Due to metabolic alterations caused by overweight, the bariatric surgery has been a largely used procedure in order to propitiate long term weight loss and maintenance in a population with morbid obesity. Objective - To analyze the impact of bariatric surgery in a population from the center area of Rio Grande do Sul State, Brazil, using the BAROS method. Method - It was carried out a retrospective study with 32 patients from both sexes, aging between 19 and 44 years old who were patients from a private clinic at the city of Santa Maria, RS. The patients' weight variation and the body mass index were analyzed, as well as their intestinal habit, use of tobacco and practice of physical activity to compare the results in pre and postoperative periods. The patients were divided into groups: group A (preoperative), group B (up to 6 months of postoperative period), group C ( 7 to 8 months of postoperative period), and group D (19 to 30 months of postoperative period). To evaluate the efficacy of the bariatric surgery BAROS method was applied. Statistical analysis was performed using analysis of variance (ANOVA), followed by post-hoc by Duncan's. It were considered as statistically significant the data with $P<0.05$. The results were expressed as mean \pm SEM Results - The patients presented a significant weight-loss, in preoperative the average was $118.8 \mathrm{~kg}( \pm 2.89)$ and, in postoperative period, group B showed $89 \mathrm{~kg}( \pm 5.69)$, group C 76.8 kg $( \pm 4.85)$ and, group D $76 \mathrm{~kg}( \pm 4.46)$, demonstrating a gradual weight loss. Likewise, the body mass index presented a significant reduction in groups B, C e D in relation to group A. The patients showed an intestinal habit improvement of $15.6 \%$ in postoperative period in relation to the preoperative period. As for the use of tobacco it was observed a decrease of $33.3 \%$ in postoperative period. The practice of a physical activity increased in $34.4 \%$ among the patients after surgery. According to the BAROS method appliance the patients showed excellent, very good and good classifications and, none of them was classified as insufficient. Conclusion - The results obtained according to the BAROS method and the patients' evaluated characteristics made it possible to observe that the bariatric surgery presented satisfactory results in this population.

HEADINGS - Obesity, morbid. Weight loss. Gastroplasty. Quality of life.

\section{REFERÊNCIAS}

1. American Society for Metabolic \& Bariatric Surgery. Rationale for the surgical treatment of morbid obesity [updted November 23, 2005]. Disponível em: www. asbms. org/Newsite07/patients/resources/asbs_rationale.htm

2. Auler Jr JOC, Giannini CG, Saragiotto DF. Desafios no manuseio peri-operatório de pacientes obesos mórbidos: como prevenir complicações. Rev Bras Anestesiol. 2003;53:227-36.

3. Brasil. Ministério da Saúde. Portaria no 628, de 26 de abril de 2001. Protocolo de indicação de tratamento cirúrgico da obesidade mórbida - Gastroplastia no âmbito do sistema único de saúde-SUS. Diário Oficial da União. Poder Executivo, Brasília, DF, 27 de abr. 2001. Disponível em: <http://www.saude.gov.br>. Acesso em: 24 jul. 2006.

4. Catafesta J. Qualidade de vida pós-cirurgia bariátrica técnica de Capella [dissertação]. Novo Hamburgo, RS: Centro Universitário Feevale; 2004.

5. Cruz MRR, Morimoto IMI. Intervenção nutricional no tratamento cirúrgico da obesidade mórbida: resultados de um protocolo diferenciado. Rev Nutr. 2004; $17: 263-72$.

6. Diniz MFFH, Diniz MTC. Obesidade mórbida. In: Amaral CFS, editor. Enciclopédia da saúde: obesidade e outros distúrbios alimentares. Rio de Janeiro: Medsi; 2002. p.290-3.

7. Fandiño J, Benchimol AK, Coutinho WF, Appolinário JC. Cirurgia bariátrica: aspectos clínico-cirúrgicos e psiquiátricos. Rev Psiquiatr Rio Gd Sul. 2004;26:4751 .

8. Faria OP, Pereira VA, Gangoni, CMC, Lins RD, Leite S, Rassi V, Arruda SLM Obesos mórbidos tratados com gastroplastia redutora com bypass gástrico em Y de Roux: análise de 160 pacientes. Brasilia Méd. 2002;39:26-34.

9. Francischi RPP, Pereira LO, Freitas CS, Klopfer M, Santos RC, Vieira P, Lanch Jr AH. Obesidade: atualização sobre sua etiologia, morbidade e tratamento. Rev Nutr. 2000;13:17-28.

10. Fujioka K. Follow-up of nutritional and metabolic problems after bariatric surgery. Diabetes Care. 2005;28:481-4.

11. Garrido Jr AB, Rodrigues JJG, Waitzberg DL. Obesidade mórbida: tratamento cirúrgico. In: Waitzberg DL, editor. Nutrição oral, enteral e parenteral na prática clínica. São Paulo: Atheneu; 2000. p.1041-7.

12. Garrido Jr AB. Cirurgia da obesidade. São Paulo: Atheneu; 2003.

13. Geloneze B, Pareja JC. Cirurgia bariátrica cura a síndrome metabólica? Arq Bras Endocrinol Metabol. 2006;50:400-7.

14. González JF, Gómez G, Arraigada G. Evaluación mediante score Baros de los resultados del bypass gástrico en el tratamiento de la obesidad mórbida. Rev Chilena Cir. 2006;58:365-70.

15. Halpern A, Segal A, Spósito AC, Ribeiro AB, Garrido A, Mady C, Fernandes F, Lorenzi-Filho G, Ramires JAF, Zanela MT, Grinberg M, Mancini M, Santos
RD. Diretrizes para cardiologistas sobre excesso de peso e doença cardiovascular dos departamentos de aterosclerose, cardiologia clínica e FUNCOR da Sociedade Brasileira de Cardiologia. Arq Bras Cardiol. 2002;78:1-13.

16. Hsu LK, Benotti PN, Dwyer J, Roberts SB, Saltzman E, Shikora S, Rolls BJ, Rand W. Nonsurgical factors that influence the outcome of bariatric surgery: a review. Psychossom Med. 1998;60:338-46.

17. James PT, Leach R, Kalamara E, Shayeghi M. The worldwide obesity epidemic. Obes Res. 2001;9(suppl 4):228s-33s.

18. Mazzeo SE, Saunders R, Mitchell KS. Binge eating among African American and Caucasian bariatric surgery candidates. Eat Behav. 2005;6:189-96.

19. Nini E, Slim K, Scesa JL, Chipponi J. Évaluation de la chirurgie coelioscopique de l'obésité par le score BAROS. An Chirurg 2002;127:107-14.

20. Oria HE, Moorehead MK. Bariatric analysis and reporting outcome system (BAROS). Obes Surg. 1998;8:487-99.

21. Paradis S, Cabanac M, Marceau P, Frankham P. Body weight and satiation after duodenal switch: 2 years later. Obes Surg. 2007;17:631-6.

22. Pareja JC, Pilla VF, Callejas-Neto F, Coelho-Neto JS, Chaim EA, Magro DO. Gastroplastia redutora com bypass gastrojejunal em Y-de-Roux: conversão para bypass gastrointestinal distal por perda insuficiente de peso - experiência em 41 pacientes. Arq Gastroenterol. 2005;42:196-200.

23. Pereira LO, Francischi RP, Lancha Jr AH. Obesidade: hábitos nutricionais, sedentarismo e resistência à insulina. Arq Bras Endocrinol Metabol. 2003;47:11127.

24. Porto MCV, Brito IC, Calfa ADF, Amoras M, Villela NB, Araújo LMB. Perfil do obeso classe III do ambulatório de obesidade de um hospital universitário de Salvador, Bahia. Arq Bras Endocrinol Metabol. 2002;46:668-73.

25. Quadros MRR, Savaris AL, Ferreira MV, Branco-Filho AJ. Intolerância alimentar no pós-operatório de pacientes submetidos a cirurgia bariátrica. Rev Bras Nutr Clin. 2007;22:15-9

26. Reis ACS, Santos AVD, Mendonça CN, Castro DSD, Hurtado JRE. Aspectos psicossociais pré e pós-cirúrgicos do paciente obeso mórbido submetido a gastroplastia redutora. Rev Horiz Cient. 2005;4:1-19.

27. Schirmer BD. Obesidade mórbida. In: Courtney MT, editor. Tratado de cirurgia: a base biológica da moderna prática cirúrgica. Rio de Janeiro: Elsevier; 2005. p.357-97.

28. Segal A, Fandiño J. Indicações e contra-indicações para realização das operações bariátricas. Rev Bras Psiq. 2002;24:68-72.

29. Velloso LA. O controle hipotalâmico da fome e da termogênese: implicações no desenvolvimento da obesidade. Arq Bras Endocrinol Metabol. 2006;50:165-76.

30. World Health Organization. Physical status: the use and interpretation of anthropometry. Genebra: World Health Organization; 1995.

Recebido em 1/7/2008 Aprovado em 2/2/2009. 